\title{
A Novel Strategy to Improve the Electrochemical Performance of a Prussian Blue Analogue Electrode for Calcium-Ion Batteries \\ Changhee LEE and Soon-Ki JEONG*
}

\author{
Department of Chemical Engineering, Soonchunhyang University, Asan, Chungnam 336-745, Republic of Korea
}

* Corresponding author: hamin611@sch.ac.kr

\begin{abstract}
Herein, mixtures of water and propylene carbonate (PC) containing $\mathrm{Ca}\left(\mathrm{CF}_{3} \mathrm{SO}_{3}\right)_{2}$ were used as electrolytes to improve the electrochemical performance parameters of a Prussian blue analogue electrode, copper hexacyanoferrate, for use in calcium-ion batteries. The performance of the electrode was greatly influenced by the molar ratio of the two solvents (water and PC) in the electrolyte. The electrode exhibited a relatively high capacity when the molar ratio of calcium cations to water in the electrolyte was 1:6. In addition, the electrode exhibited a coulombic efficiency of $\sim 100 \%$ over 800 cycles, with $\sim 94 \%$ retention of the maximum capacity after 800 cycles in this electrolyte solution. Raman spectroscopy revealed that calcium cations were preferentially solvated by water molecules in the electrolyte, which is believed to be related to the improvement in electrode performance.
\end{abstract}

(c) The Electrochemical Society of Japan, All rights reserved.

Keywords : Calcium-ion Batteries, Prussian Blue Analogue, Propylene Carbonate, Raman Spectroscopy

\section{Introduction}

Studies on new secondary battery systems that could replace lithium-ion batteries have recently begun to attract attention. ${ }^{1-4}$ Calcium-ion batteries (CIBs), which are polyvalent-ion batteries, have been investigated as a potential alternative to lithium-ion batteries. In principle, CIBs are expected to have relatively high capacities because calcium cations undergo two electronic reactions during redox processes. ${ }^{5-8}$ Additionally, calcium is the fifth most abundant element on Earth. These advantages have led to the expectation that CIBs will have high energy densities and low costs. ${ }^{9-13}$ However, one major problem of CIBs is that calcium cations diffuse very slowly in electrodes owing to their high charge density, resulting in poor electrochemical performance; this problem is also present in other polyvalent-ion batteries. ${ }^{14-16}$ Recently, it has been demonstrated that this problem can be resolved by using aqueous electrolytes. ${ }^{17,18}$ As polyvalent ions are hydrated (solvated by water molecules) in aqueous electrolytes, their charge densities are reduced by the shielding effects of water, enabling their rapid diffusion in the electrode. In such cases, the use of electrode materials with large channels that allow the diffusion of hydrated cations is necessary.

Several researchers have observed that calcium cations can rapidly diffuse in aqueous rechargeable systems using Prussian blue analogue (PBA) electrodes, which have large open interstitial sites and $3 \mathrm{D}$ open channels that allow ionic diffusion within the cyanobridged perovskite framework. ${ }^{19,20}$ However, in such systems, improvement of various electrochemical performance parameters, including the capacity reduction during the initial cycles and the relatively low discharge/charge capacities, is still required. In a previous study, we reported that the electrochemical performance could be improved by controlling the hydration number of calcium cations in an aqueous electrolyte through the application of a superconcentrated electrolyte in a copper hexacyanoferrate $\left(\mathrm{CuFe}(\mathrm{CN})_{6}\right.$, $\mathrm{CuHCF}$ ) electrode, which is one of the PBAs used in CIBs. ${ }^{21} \mathrm{We}$ found that the discharge/charge capacities and cycling performance of the $\mathrm{CuHCF}$ electrode could be improved, which might be because of the decreased hydration number of calcium ions, when applied the superconcentrated aqueous electrolyte. However, the superconcentrated electrolyte had some limitations, such as the use of excess salt and a high viscosity, which are unfavorable properties for battery electrolytes. ${ }^{22}$

In this study, mixtures of water and propylene carbonate (PC) containing $\mathrm{Ca}\left(\mathrm{CF}_{3} \mathrm{SO}_{3}\right)_{2}$ (hereinafter referred to as $\mathrm{Ca}-\mathrm{WP}$ electrolytes) were used as a novel strategy to improve the electrochemical performance of the CuHCF electrode for CIBs. According to studies on donor number (DN), which is a quantitative measure of Lewis basicity, the DN of water (33) is higher than that of PC (15.1). ${ }^{23,24}$ It is well known that solvent molecules with higher DNs exhibit stronger cation solvation in an electrolyte. ${ }^{25}$ Additionally, in previous studies on the solvation of cations, it has been reported that hydration enthalpies with water molecules are lower than solvation enthalpies with PC molecules. ${ }^{26-28}$ Therefore, in Ca-WP electrolytes, calcium cations may be primarily solvated by water molecules, not by PC. In addition, the presence of excess PC in Ca-WP electrolytes may reduce the hydration number of calcium cations. Based on this assumption, we conducted studies with the expectation that the Ca-WP electrolytes would overcome the limitations of the aqueous electrolytes mentioned above, thereby improving the electrode performance.

\section{Experimental}

\subsection{Electrode and electrolytes}

The PBA used as an active material in this system was CuHCF. $\mathrm{CuHCF}$ powder was synthesized according to the method reported by Cui et al. ${ }^{19,20}$ In brief, $120 \mathrm{~cm}^{3}$ of $0.1 \mathrm{~mol} \mathrm{dm}^{-3} \mathrm{Cu}\left(\mathrm{NO}_{3}\right)_{2}$ (Alfa Aesar, $99.999 \%$ ) and $120 \mathrm{~cm}^{3}$ of $0.05 \mathrm{~mol} \mathrm{dm}^{-3} \mathrm{~K}_{3} \mathrm{Fe}(\mathrm{CN})_{6}$ (Sigma Aldrich, 99\%) were simultaneously added in a dropwise manner to $60 \mathrm{~cm}^{3}$ of deionized (DI) water (Burdick \& Jackson, HPLC grade) under constant stirring at $80^{\circ} \mathrm{C}$. After sonicating for $30 \mathrm{~min}$, the suspension was aged for $6 \mathrm{~h}$ at $80^{\circ} \mathrm{C}$. The precipitate was filtered and dried under vacuum at room temperature for $24 \mathrm{~h}$. The structural analysis of the synthesized CuHCF powder can be found in our previous study. ${ }^{21}$

To prepare the $\mathrm{CuHCF}$ electrode, a slurry containing a mixture of the synthesized $\mathrm{CuHCF}$ powder $(80 \mathrm{wt} \%)$, Super $\mathrm{P}(9 \mathrm{wt} \%)$, graphite powder $(2 \mathrm{wt} \%)$, and polyvinylidene fluoride $(9 \mathrm{wt} \%$, Sigma Aldrich) was made and coated on a carbon cloth (Fuel Cell Earth) that acted as a current collector. The coated electrode was dried 

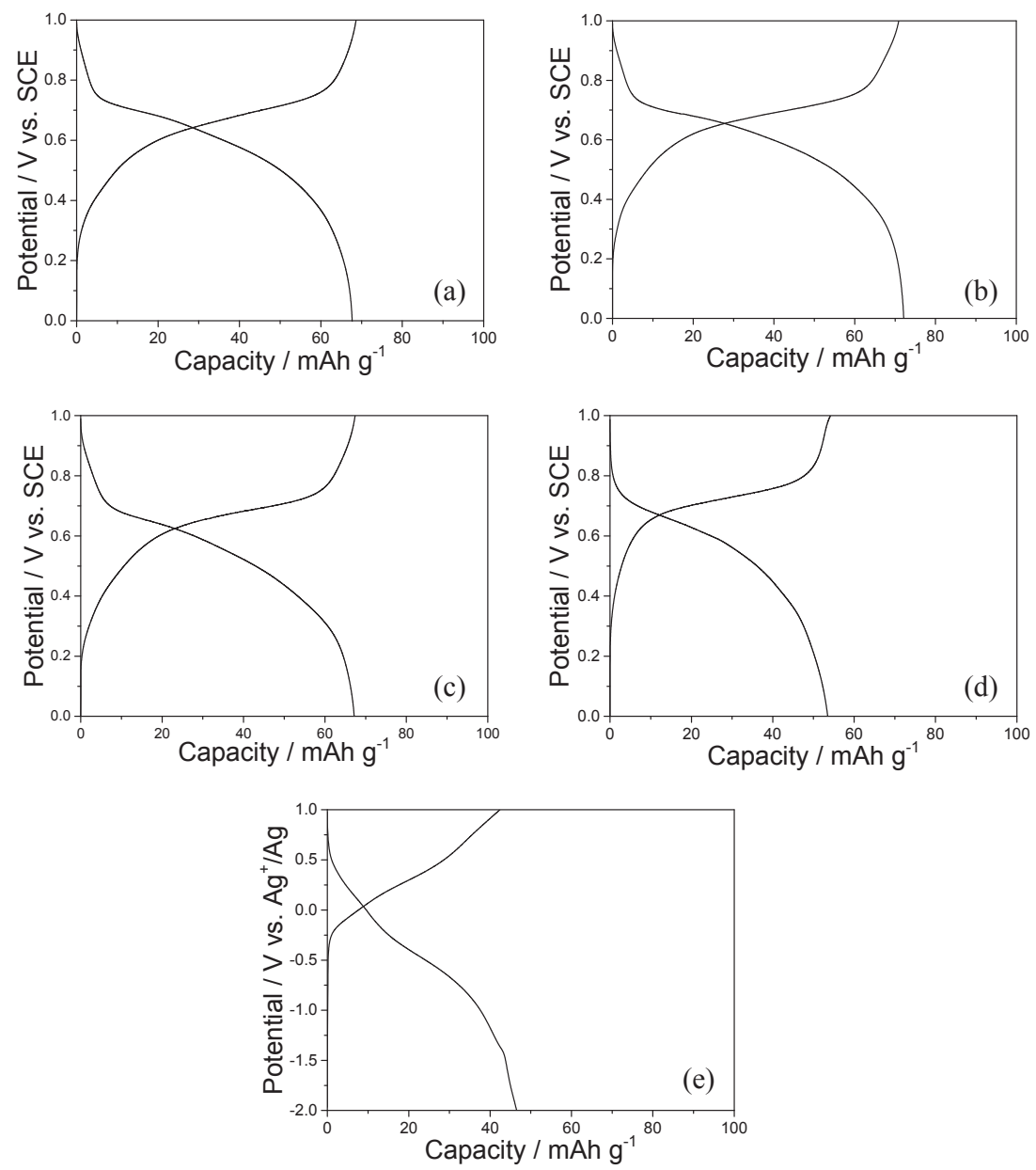

Figure 1. Discharge/charge curves of CuHCF electrodes in (a) Ca-WP12, (b) Ca-WP6, (c) Ca-WP5, (d) Ca-WP3, and (e) Ca-PC electrolytes.

under vacuum at $80^{\circ} \mathrm{C}$ for $12 \mathrm{~h}$ to obtain the desired electrode. Prior to electrochemical tests, the $\mathrm{CuHCF}$ electrode was charged from the open circuit voltage to $1 \mathrm{~V}$ vs. saturated calomel electrode (SCE) in DI water over $12 \mathrm{~h}$ to extract $\mathrm{K}^{+}$ions. The electrode was then rinsed with DI water and dried at $60^{\circ} \mathrm{C}$ for $12 \mathrm{~h}$.

The Ca-WP electrolytes were prepared by dissolving $0.1 \mathrm{~mol} \mathrm{dm}^{-3} \mathrm{Ca}\left(\mathrm{CF}_{3} \mathrm{SO}_{3}\right)_{2}$ (Sigma Aldrich, 99.9\%) in mixtures of water and PC (Enchem, battery grade). The molar ratios of calcium cations to water in the electrolytes were $1: 12,1: 6,1: 5$, and 1:3, which correspond to molar ratios of water to PC of 1:9.8, 1:19.5, 1:23.4, and 1:33.1. These electrolytes were named Ca-WP12, Ca-WP6, Ca-WP5, and Ca-WP3, respectively. For comparison with the $\mathrm{Ca}-W \mathrm{P}$ electrolytes, $0.1 \mathrm{moldm}^{-3} \mathrm{Ca}\left(\mathrm{CF}_{3} \mathrm{SO}_{3}\right)_{2}$ in $\mathrm{PC}$ without any water (named $\mathrm{Ca}-\mathrm{PC}$ ) was prepared. Before dissolution, the $\mathrm{Ca}\left(\mathrm{CF}_{3} \mathrm{SO}_{3}\right)_{2}$ salt was dried under vacuum for $24 \mathrm{~h}$ at room temperature.

The Raman spectra of the electrolyte solutions were recorded using a DeltaNu/NS785 spectrometer. Excitation was carried out with a $785 \mathrm{~nm}(120 \mathrm{~mW})$ laser.

\subsection{Electrochemical tests}

Electrochemical tests in the Ca-WP electrolytes were carried out by using a three-electrode cell at room temperature. SCE and activated carbon were used as the reference and counter electrodes, respectively. In addition, electrochemical tests in the $\mathrm{Ca}-\mathrm{PC}$ electrolyte were performed by using a three-electrode cell in an argon-filled glovebox (Three Shine, SK-G1200) with a dew point lower than $-60^{\circ} \mathrm{C}$. Silver wire and activated carbon were used as the reference and counter electrodes, respectively. In the electrochemical test cell, the loading mass and geometric area of the CuHCF electrode were $0.0038 \mathrm{~g} \mathrm{~cm}^{-1}$ and $0.56 \mathrm{~cm}^{2}$, respectively, and the amount of electrolyte solution was $25 \mathrm{~cm}^{3}$. The tests were performed on a battery test system (WonATech, WBCS3000). The discharge/ charge and cyclability tests were carried out at $0.2 \mathrm{C}$ and $2 \mathrm{C}$, respectively.

\section{Results and Discussion}

First, discharge/charge tests were performed in the Ca-WP electrolytes to investigate the effects of excess $\mathrm{PC}$ on the electrochemical performance of the $\mathrm{CuHCF}$ electrode. In previous studies on aqueous electrolytes, it has been reported that calcium cations can have a hydration number of up to nine for the first shell if there are sufficient water molecules in the electrolyte, although there is some variation in this value depending on the analysis method. ${ }^{29-31}$ Based on this, the discharge/charge tests were carried out in $\mathrm{Ca}-W P$ electrolytes with $\mathrm{H}_{2} \mathrm{O} / \mathrm{Ca}^{2+}$ molar ratios of more than or less than nine to clarify the effect of decreasing the hydration number of calcium ions.

A comparison of Figs. 1a and $1 \mathrm{~b}$ shows that the reversible discharge/charge capacities increased slightly in the Ca-WP6 electrolyte relative to those in the Ca-WP12 electrolyte. The capacities in the Ca-WP6 electrolyte were $\sim 12 \%$ higher than those obtained in a dilute aqueous electrolyte in our previous study. ${ }^{21}$ Owing to sufficient amounts of water in the electrolyte, the calcium cations in the Ca-WP12 and dilute aqueous electrolyte will have large hydration numbers. However, as there are fewer water molecules in the Ca-WP6 electrolyte, calcium cations in this 
electrolyte may have a smaller hydration number than those in the Ca-WP12 and dilute aqueous electrolyte. Therefore, the increased reversible discharge/charge capacities in the Ca-WP6 electrolyte could be due to the reduced hydration number of the calcium cations, which enabled the hydrated calcium cations to be inserted into/extracted from the $\mathrm{CuHCF}$ electrode more easily.

In contrast, on increasing the amount of $\mathrm{PC}$ in the electrolyte relative to that in the Ca-WP6 electrolyte, the discharge/charge capacities gradually decrease (Figs. 1c-1e). In particular, the lowest discharge/charge capacities were observed in the Ca-PC electrolyte. This result is believed to be due to the interaction of PC with the calcium cations caused by a lack of water molecules, with this interaction becoming stronger as the amount of PC in the electrolyte increases. Calcium cations that interact with large PC molecules might be difficult to insert into/extract from the $\mathrm{CuHCF}$ electrode, resulting in relatively poor discharge/charge capacities.

Hereinafter, only results obtained using the Ca-WP6 electrolyte will be described, as the CuHCF electrode exhibited the best discharge/charge performance in this electrolyte. As mentioned, calcium cations were expected to be preferentially hydrated in the Ca-WP electrolytes because water has a higher DN and a lower solvation enthalpy for cations than PC. Thus, we investigated whether calcium cations were solvated by water or PC molecules in the Ca-WP6 electrolyte by analyzing the hydration or solvation state of the cations using Raman spectroscopy. In general, cation-PC interactions are identified using the skeletal bending mode of $\mathrm{PC}$ at $712 \mathrm{~cm}^{-1}$ in Raman spectroscopy, which has been mainly used in $\mathrm{Li}^{+}-\mathrm{PC}$ solvation studies. ${ }^{32,33}$ As it is well known that the peak at $712 \mathrm{~cm}^{-1}$ is shifted to higher wavenumbers by $\mathrm{Li}^{+}-\mathrm{PC}$ interactions, we considered that $\mathrm{Ca}^{2+}-\mathrm{PC}$ interactions could be analyzed through an analogous shift. However, it was difficult to analyze the solvation of calcium cations by PC molecules or water molecules in the Ca-WP6 electrolyte using the peak at $712 \mathrm{~cm}^{-1}$ owing to the $\mathrm{v}_{l 2}$ librational band of water, which is reported to appear at $\sim 700$ $750 \mathrm{~cm}^{-1} \cdot 34,35$ Therefore, the solvation state of calcium cations in the electrolytes was discussed by examining the Raman peak at $\sim 848 \mathrm{~cm}^{-1}$.

As shown in Fig. 2a, a strong peak is observed at $\sim 848 \mathrm{~cm}^{-1}$ in pure $\mathrm{PC}$ solution originating from the symmetric ring vibration of PC molecules. ${ }^{36}$ In addition to this peak, a new peak is observed at $\sim 859 \mathrm{~cm}^{-1}$ in the Ca-PC electrolyte (Fig. $2 \mathrm{~b}$ ), indicating that some calcium cations are solvated by PC molecules. In other words, the peaks at $\sim 848$ and $\sim 859 \mathrm{~cm}^{-1}$ originate from free and solvated PC molecules, respectively. However, the peak at $\sim 859 \mathrm{~cm}^{-1}$ is not observed in the Ca-WP6 electrolyte containing both PC and water molecules (Fig. 2c), indicating that PC molecules do not participate in solvation and calcium cations are preferentially hydrated in this

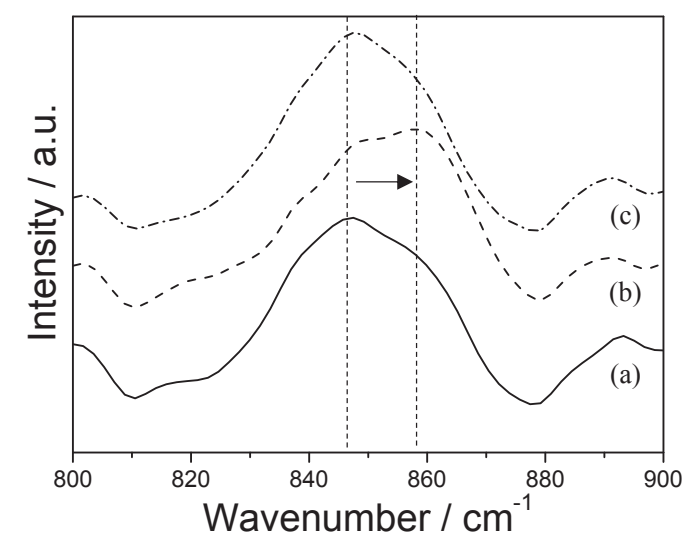

Figure 2. Raman spectra of (a) pure PC solution, (b) Ca-PC, and (c) Ca-WP6 electrolytes.

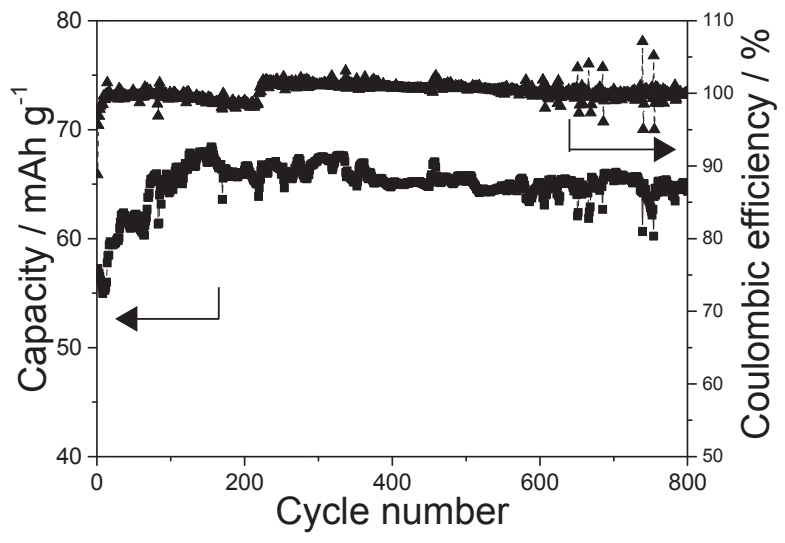

Figure 3. Cyclability and coulombic efficiency of the $\mathrm{CuHCF}$ electrode in the Ca-WP6 electrolyte.

electrolyte. This result suggests that the degree of hydration of calcium cations in Ca-WP electrolytes, which affects the discharge/ charge performance, can be controlled to some extent by adjusting the ratio of water to $\mathrm{PC}$ in the electrolyte where the concentration of calcium salt is fixed.

Additional electrochemical measurements were performed to investigate the physicochemical properties of the Ca-WP6 electrolyte. As shown in Fig. 3, the CuHCF electrode exhibited excellent cyclability and columbic efficiency over 800 cycles in the Ca-WP6 electrolyte. In our previous work, we demonstrated through X-ray diffraction analysis that capacity reduction of the $\mathrm{CuHCF}$ electrode in aqueous electrolyte systems mainly resulted from structural collapse of the CuHCF material. ${ }^{21}$ The structural collapse after 150 cycles was attributed primarily to repeated insertion/extraction of calcium cations with relatively large hydration numbers and radii in aqueous electrolytes with sufficient amounts of water molecules. Therefore, the excellent cyclability and columbic efficiency observed in the Ca-WP6 electrolyte could be attributed to the presence of calcium cations with relatively small hydration numbers and radii. Such cations could be inserted into/extracted from the $\mathrm{CuHCF}$ material repeatedly and smoothly, without significant structural stress on the CuHCF electrode. Notably, the charge capacity gradually increased over 200 cycles. Unfortunately, the origin of this unusual phenomenon is unclear, although similar activation behavior has been reported for other systems. ${ }^{37,38}$ Regardless, a detailed investigation of this phenomenon is beyond the scope of the present work.

Figure $4 \mathrm{a}$ shows the rate capability of the $\mathrm{CuHCF}$ electrode in the Ca-WP6 electrolyte. The results obtained in the Ca-PC electrolyte without water are shown in Fig. $4 \mathrm{~b}$ for comparison. A charge capacity retention of $\sim 52 \%$ at $10 \mathrm{C}$ was observed in the Ca-WP6 electrolyte, whereas a value of $\sim 25 \%$ at $10 \mathrm{C}$ was observed in the Ca-PC electrolyte. The improved rate capability achieved in the Ca-WP6 electrolyte may result from hydration of the calcium cations in the electrolyte, which enables smoother insertion/ extraction and rapid diffusion of calcium cations into the electrode owing to the effects of water shielding.

\section{Conclusions}

The performance parameters (capacity, cyclability, and rate capability) of a CuHCF electrode were improved by using a new electrolyte system consisting of water, PC, and calcium salt (Ca-WP electrolytes). Compared with an aqueous electrolyte containing no PC (Ca-PC electrolyte), significantly improved performance parameters were observed in the Ca-WP6 electrolyte (molar ratio of calcium cations to water of $1: 6)$ because the solvation structure of 

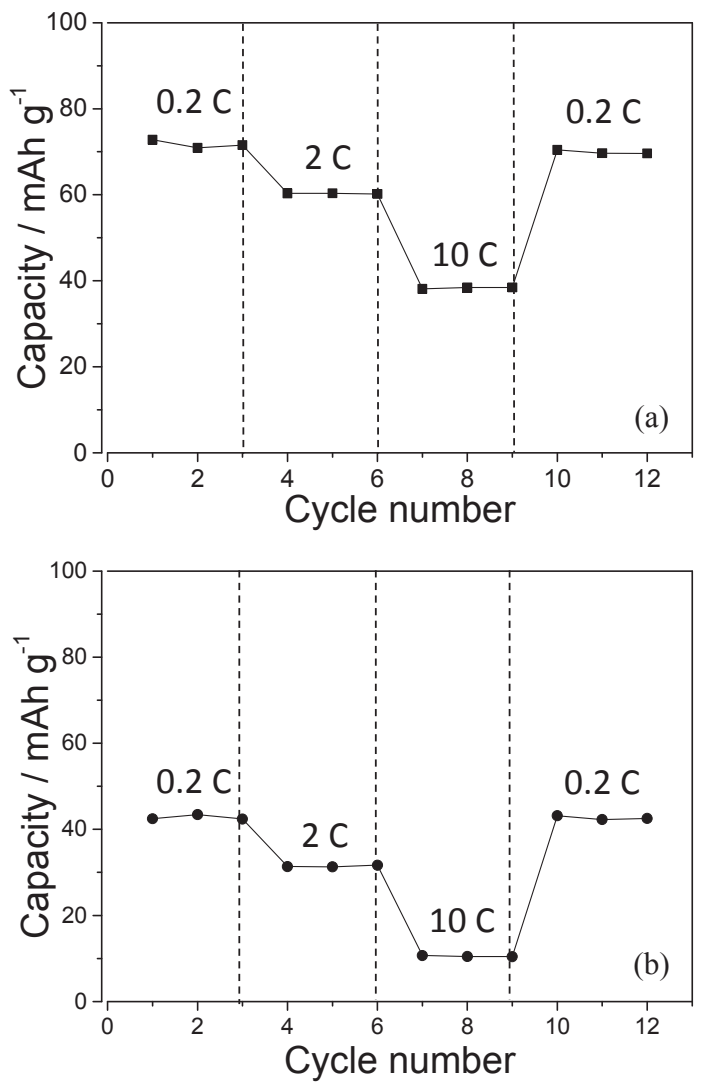

Figure 4. Rate capabilities of the CuHCF electrode in (a) Ca-WP6 and (b) Ca-PC electrolytes.

the calcium cations was altered by the presence of PC. Analysis of the Raman spectra of the electrolytes revealed that PC played a role in reducing the number of water molecules solvating the calcium cations without directly participating in solvation. The performance of the CuHCF electrode was highly dependent on the amount of PC in the electrolyte. This study suggests that the performance of the $\mathrm{CuHCF}$ electrode can be improved by using a small amount of water, which induces a shielding effect, in combination with a large amount of solvent that is only capable of weak interactions with calcium cations in CIBs.

\section{Acknowledgments}

This research was supported by the Basic Science Research Program through the National Research Foundation of Korea (NRF) funded by the Ministry of Education (No. NRF2017R1A2B4010544). This work was also supported by the Soonchunhyang University Research Fund.

\section{References}

1. R. E. Doe, R. Han, J. Hwang, A. J. Gmitter, I. Shterenberg, H. D. Yoo, N. Pour, and D. Aurbach, Chem. Commun., 50, 243 (2014).

2. H. D. Yoo, I. Shterenberg, Y. Gofer, G. Gershinsky, N. Pour, and D. Aurbach, Energy Environ. Sci., 6, 2265 (2013).

3. J. Muldoon, C. B. Bucur, A. G. Oliver, T. Sugimoto, M. Matsui, H. S. Kim, G. D. Allred, J. Zajicek, and Y. Kotani, Energy Environ. Sci., 5, 5941 (2012).

4. N. Wu, Z.-Z. Yang, H.-R. Yao, Y.-X. Yin, L. Gu, and Y.-G. Guo, Angew. Chem., Int. Ed., 54, 5757 (2015)

5. G. G. Amatucci, F. Badway, A. Singhal, B. Beaudoin, G. Skandan, T. Bowmer, I. Plitz, N. Pereira, T. Chapman, and R. Jaworski, J. Electrochem. Soc., 148, A940 (2001).

6. E. Levi, M. D. Levi, O. Chasid, and D. Aurbach, J. Electroceram., 22, 13 (2009).

7. D. Aurbach, I. Weissman, Y. Gofer, and E. Levi, Chem. Rec., 3, 61 (2003).

8. N. Singh, T. S. Arthur, C. Ling, M. Matsui, and F. Mizuno, Chem. Commun., 49, 149 (2013).

9. N. Emery, C. Hérold, and P. Lagrange, J. Solid State Chem., 178, 2947 (2005).

10. M. Hayashi, H. Arai, H. Ohtwuka, and Y. Sakurai, J. Power Sources, 119-121, 617 (2003).

11. T. E. Weller, M. Ellerby, S. S. Saxena, R. P. Smith, and N. T. Skipper, Nat. Phys., 1, 39 (2005).

12. M. Smeu, M. S. Hossain, Z. Wang, V. Timoshevskii, K. H. Bevan, and K. Zaghib, J. Power Sources, 306, 431 (2016).

13. M. Cabello, F. Nacimiento, J. R. González, G. Ortiz, R. Alcántara, P. Lavela, C. Pérez-Vicente, and J. L. Tirado, Electrochem. Commun., 67, 59 (2016).

14. E. Levi, Y. Gofer, and D. Aurbach, Chem. Mater, 22, 860 (2010).

15. Y. Liang, R. Feng, S. Yang, H. Ma, J. Liang, and J. Chen, Adv. Mater, 23, 640 (2011).

16. D. Aurbach, G. S. Suresh, E. Levi, A. Mitelman, O. Mizrahi, O. Chusid, and M. Brunelli, Adv. Mater. 19, 4260 (2007).

17. J. Song, M. Noked, E. Gillette, J. Duay, G. Rubloff, and S. B. Lee, Phys. Chem. Chem. Phys., 17, 5256 (2015).

18. R. K. Guduru and J. C. Icaza, Nanomaterials (Basel), 6, 41 (2016)

19. R. Y. Wang, B. Shyam, K. H. Stone, J. N. Weker, M. Pasta, H.-W. Lee, M. F. Toney, and Y. Cui, Adv. Energy Mater., 5, 1401869 (2015).

20. R. Y. Wang, C. D. Wessells, R. A. Huggins, and Y. Cui, Nano Lett., 13, 5748 (2013).

21. C. H. Lee and S.-K. Jeong, Chem. Lett., 45, 1447 (2016).

22. Y. Yamada and A. Yamada, J. Electroanal. Chem., 162, A2406 (2015).

23. V. Gutmann, Electrochim. Acta, 21, 661 (1976).

24. C. S. Kim and S. M. Oh, Electrochim. Acta, 45, 2101 (2000).

25. S.-I. Tobishima and A. Yamaji, Electrochim. Acta, 29, 267 (1984).

26. D. W. Smith, J. Chem. Educ., 54, 540 (1977).

27. Y. Yamada, Y. Takazawa, K. Miyazaki, K. Miyazaki, and T. Abe, J. Phys. Chem. $C, \mathbf{1 1 4}, 11680$ (2010).

28. M. Shakourian-Fard, G. Kamath, K. Smith, H. Xiong, and S. K. R. S. Sankaranarayanan, J. Phys. Chem. C, 119, 22747 (2015).

29. P. Bopp, Pure Appl. Chem., 59, 1071 (1987).

30. J. Lindgren and K. Hermansson, J. Phys. Chem., 97, 5254 (1993).

31. T. Oi, K. Sato, and K. Umemoto, Z. Naturforsch., A: Phys. Sci., 68, 362 (2013).

32. Z. Wang, W. Gao, X. Huang, Y. Mo, and L. Chen, J. Raman Spectrosc., 32, 900 (2001).

33. S.-K. Jeong, M. Inaba, Y. Iriyama, T. Abe, and Z. Ogumi, Electrochem. Solid-State Lett., 6, A13 (2003).

34. G. E. Walrafen, J. Chem. Phys., 47, 114 (1967).

35. G. E. Walrafen, J. Chem. Phys., 40, 3249 (1964)

36. G. J. Janz, J. Ambrose, J. W. Coutts, and J. R. Downey, Spectrochim. Acta, Part A, 35, 175 (1979).

37. C. J. Barile, E. C. Barile, K. R. Zavadil, R. G. Nuzzo, and A. A. Gewirth, J. Phys. Chem. C, 118, 27623 (2014).

38. Z. Hou, X. Zhang, X. Li, Y. Zhu, J. Liang, and Y. Qian, J. Mater. Chem. A, 5, 730 (2017). 